\title{
Epidemiology of Liver Cirrhosis
}

\section{Dear Editor,}

Liver cirrhosis is a major public health problem and a significant source of morbidity and mortality that is preventable and underestimated. ${ }^{1}$ It represents the main indication for liver transplantation in both United States and Europe. ${ }^{2}$

Recently in a large secondary care hospital, Hsiang et $\mathrm{al},{ }^{3}$ showed that the common primary aetiologies for liver cirrhosis were chronic hepatitis B cirrhosis (37.3\%), alcoholic liver disease cirrhosis (24.1\%), chronic hepatitis C cirrhosis $(22.3 \%)$ and non-alcoholic fatty liver disease cirrhosis (16.4\%).

All chronic liver diseases are characterized by their capacity to progress into cirrhosis. The fibrogenic process goes through various phases where excessive collagen deposition results in qualitative and quantitative changes of the extracellular matrix. Cirrhosis is the structural subversion of the liver with the formation of regenerative nodules and it represents the late-stage liver disease. Advanced cirrhosis is a condition with limited treatment options.

Changes in mortality for cirrhosis in different countries reflect differences in the prevalence of risk factors such as alcohol abuse and HBV and HCV infection. ${ }^{4}$ In 2010, liver cirrhosis was the 23 rd cause of death worldwide (31 million), Disability Adjusted Life Years with roughly equal proportions attributable to $\mathrm{HCV}, \mathrm{HBV}$ and alcohol consumption. ${ }^{5}$

Although cirrhosis is predominantly of viral etiology, with the current encoding system it is not possible to identify cases of cirrhosis of viral etiology. In fact, the International Classification of Diseases (ICD) permits assignment of an ICD code to the underlying cause of death as noted in a death certificate. Depending on information available on the death, a more general code can only be assigned, for example, unspecified cirrhosis of the liver. However, some observations can be made. The use of this code could underestimate the true incidence rate for cirrhotic patients who die. For example, deaths for hepatorenal syndrome or portal hypertension are currently classified with other codes, because the classification is based only on the main cause of death. As previously mentioned, a further issue is the lack of a subcategory that identifies cirrhosis, such as viral etiology. This suggests that the mortality rate tends to underestimate the illnesses that are really incident to cirrhosis.
In one of the main global studies on liver cirrhosis mortality performed in 187 countries between 1980 and 2010, unfortunately, there were no data available from Central Sub-Saharan Africa and verbal autopsy comprised the majority of information accrued for Eastern and Western Sub-Saharan Africa. Therefore no information was available for $31 \%$ of countries. ${ }^{4}$ This confirms that the global burden of liver cirrhosis was underestimated.

\section{CONFLICTS OF INTEREST}

The authors have none to declare.

\section{REFERENCES}

1. Blachier M, Leleu H, Peck-Radosavljevic M, Valla DC, Roudot-Thoraval F. The burden of liver disease in Europe: a review of available epidemiological data. J Hepatol. 2013;58:593-608.

2. Burra P. Hepatitis C. Semin Liver Dis. 2009;29:53-65.

3. Hsiang JC, Bai WW, Raos Z, et al. Epidemiology, disease burden and outcomes of cirrhosis in a large secondary care hospital in South Auckland, New Zealand. Intern Med J. 2015;45:160-169.

4. Mokdad AA, Lopez AD, Shahraz S, et al. Liver cirrhosis mortality in 187 countries between 1980 and 2010: a systematic analysis. BMC Med. 2014 Sep 18;12(1):145.

5. Murray CJ, Vos T, Lozano R, et al. Disability-adjusted life years (DALYs) for 291 diseases and injuries in 21regions, 1990-2010: a systematic analysis for the Global Burden of Disease Study 2010. Lancet. 2012;380:2197-2223.

\section{Cristina Stasi}

Health Agency of Tuscany, Florence, Italy and Department of Experimental and Clinical Medicine, University of Florence, Florence, Italy

\section{Caterina Silvestri, Fabio Voller, Francesco Cipriani} Health Agency of Tuscany, Florence, Italy

Address for correspondence: Cristina Stasi, Health Agency of Tuscany, Florence, Italy. Tel.: +39 055 4624385; fax: +390554624330 .

E-mail: cristina.stasi@gmail.com

15 May 2015

Available online: 14 June 2015 